\title{
Regional lung ventilation estimation based on supervoxel tracking
}

\section{Adam Szmul, Bartlomiej W. Papiez, Tahreema Matin, Fergus V. Gleeson, Julia A. Schnabel, et al.}

Adam Szmul, Bartlomiej W. Papiez, Tahreema Matin, Fergus V. Gleeson, Julia A. Schnabel, Vicente Grau, "Regional lung ventilation estimation based on supervoxel tracking," Proc. SPIE 10576, Medical Imaging 2018: ImageGuided Procedures, Robotic Interventions, and Modeling, 105761E (13 March 2018); doi: 10.1117/12.2293833

SPIE. Event: SPIE Medical Imaging, 2018, Houston, Texas, United States 


\title{
Regional Lung Ventilation Estimation Based on Supervoxel Tracking
}

\author{
Adam Szmul ${ }^{\mathrm{a}}$, Bartłomiej W. Papież ${ }^{\mathrm{a}}$, Tahreema Matin ${ }^{\mathrm{b}}$, Fergus V. Gleeson ${ }^{\mathrm{b}, \mathrm{c}}$, Julia A. \\ Schnabel ${ }^{\mathrm{a}, \mathrm{d}}$, and Vicente $\mathrm{Grau}^{\mathrm{a}}$ \\ ${ }^{a}$ Institute of Biomedical Engineering, Department of Engineering Science, University of \\ Oxford, UK, \\ ${ }^{\mathrm{b}}$ Department of Oncology, University of Oxford, UK \\ 'Department of Radiology, Oxford University Hospitals NHS FT, Oxford, UK \\ ${ }^{\mathrm{d}}$ Department of Biomedical Engineering, School of Biomedical Engineering and Imaging \\ Sciences, Kings College London, UK
}

\begin{abstract}
In the case of lung cancer, an assessment of regional lung function has the potential to guide more accurate radiotherapy treatment. This could spare well-functioning parts of the lungs, as well as be used for follow up. In this paper we present a novel approach for regional lung ventilation estimation from dynamic lung CT imaging, which might be used during radiotherapy planning. Our method combines a supervoxel-based image representation with deformable image registration, performed between peak breathing phases, for which we track changes in intensity of previously extracted supervoxels. Such a region-oriented approach is expected to be more physiologically consistent with lung anatomy than previous methods relying on voxel-wise relationships, as it has the potential to mimic the lung anatomy. Our results are compared with static ventilation images acquired from hyperpolarized Xenon129 MRI. In our study we use three patient datasets consisting of 4DCT and XeMRI. We achieve higher correlation (0.487) compared to the commonly used method for estimating ventilation performed in a voxel-wise manner (0.423) on average based on global correlation coefficients. We also achieve higher correlation values for our method when ventilated/non-ventilated regions of lungs are investigated. The increase of the number of layers of supervoxels further improves our results, with one layer achieving 0.393, compared to 0.487 for 15 layers. Overall, we have shown that our method achieves higher correlation values compared to the previously used approach, when correlated with XeMRI.
\end{abstract}

Keywords: supervoxels, tracking, lung ventilation estimation, image registration

\section{INTRODUCTION}

Pulmonary-related diseases are among the leading causes of death worldwide, ${ }^{1}$ with lung cancer being the most common cause of cancer death and the second most commonly diagnosed cancer type in both male and female populations. ${ }^{2}$ Global measurements of lung functionality do not give enough information about the local condition of the lungs. An assessment of the regional lung functionality has the potential to provide more accurate radiotherapy treatment, which could spare well-functioning parts of the lungs, as well as be used for follow up.

An imaging technique that has recently received substantial attention in the field is hyperpolarized noble gas MRI. ${ }^{3}$ Its non-ionizing nature makes it a promising technique for imaging ventilation, perfusion and gas transfer within the lungs. ${ }^{4}$ In this method, the patient breathes in a fixed volume of hyperpolarized noble gas and images are acquired during breath hold. Hyperpolarized Xenon-129 MRI (XeMRI) provides images with direct visualization of the distribution of inhaled gas in the patient's lungs. Compared to other lung functional imaging techniques, XeMRI images are acquired during a single breath-hold. To allow for the localization of ventilation images with respect to the patient's lung boundaries, proton density MRI (pMRI) is acquired immediately

A.S.: E-mail: adam.szmul@eng.ox.ac.uk, Telephone: +44 01865617675

Medical Imaging 2018: Image-Guided Procedures, Robotic Interventions, and Modeling, edited by Baowei Fei, Robert J. Webster III, Proc. of SPIE Vol. 10576, 105761E · C 2018 SPIE

CCC code: $1605-7422 / 18 / \$ 18 \cdot$ doi: $10.1117 / 12.2293833$

Proc. of SPIE Vol. 10576 105761E-1 
before XeMRI acquisition. Currently, the main drawback of the technique is its accessibility, which is limited to a number of imaging centers in the world.

4DCT, on the other hand, is a structural imaging technique used for radiotherapy planning, where images are acquired during free breathing. It can be used to estimate lung ventilation maps, based on the results of the registration between peak-exhale and peak-inhale breathing phase volumes. 4DCT based ventilation methods can be roughly classified into those which rely on the value of the determinant of the Jacobian matrix of the deformation field to estimate the lung volume change, ${ }^{5}$ and methods tracking the changes in the intensity of the lungs, quantified in Hounsfield units $(\mathrm{HU})^{6,7}$. Changes in tissue attenuation correspond to variations in tissue density and originate from filling the lungs with air. In our study we focus on this methodology, as it has been shown to correlate well with lung function in emphysema patients ${ }^{8}$.

\section{METHODS}

In this section we present the proposed supervoxel-based image clustering method and explain its application to estimate lung ventilation using deformations estimated by deformable image registration between peak breathing phase volumes.

\subsection{Lung Image Registration}

The proposed method requires estimation of the deformation field between peak exhale and peak inhale lung images from 4DCT. As the images are already rigidly aligned due to their acquisition set-up, we applied only deformable image registration to estimate deformations $\mathbf{u}_{e x h}$. To this end we used the deeds method, ${ }^{9}$ which has been reported to achieve high performance in $4 \mathrm{DCT}$ lung image registration. ${ }^{10}$ To compare the estimated ventilation images with XeMRI ventilation maps, we need to find a mapping from XeMRI to CT images. In the comparison, we use the peak inhale $4 \mathrm{DCT}$ phase, when the lung volume is more consistent with pMRI and XeMRI, which are acquired after inhalation of 1 liter of gas. This makes the comparison more reliable, as the images will undergo smaller deformations.

XeMRI images were aligned with peak inhale 4DCT phase using pMRI to CT registration as an intermediate step. To achieve that, we applied rigid registration with mutual information as a similarity measure followed by deformable image registration also using deeds. ${ }^{9}$ The analysis of the influence of the registration method is beyond the scope of this study.

\subsection{Supervoxel Clustering}

Supervoxel-based image clustering groups voxels that are spatially close and visually similar into larger structures. They have become building blocks for many applications in computer vision and medical image analysis. In our formulation, we apply Simple Linear Iterative Clustering (SLIC) ${ }^{11}$ as a clustering method, due to its speed of performance and direct control over the number of extracted supervoxels and supervoxel compactness. The SLIC method is designed to extract $k$ approximately equally-sized supervoxels. Initially, seeds of the supervoxels are placed on a regular grid at intervals $S=\sqrt[3]{M / k}$ voxels apart, with $M$ being the total number of voxels in the image. In the original work by Achanta, ${ }^{11}$ the method was proposed for $2 \mathrm{D}$ images, whereas here we apply it to 3D supervoxels. The positions of the centers are then corrected based on the gradients of the image to prevent from placing them on image edges or at a noisy voxel. Individual voxels are assigned to the closest cluster based on the distance

$$
\Upsilon=\sqrt{\left(d_{e}\right)^{2}+\left(\frac{d_{I}}{S}\right)^{2} m^{2}},
$$

where $m$ is a parameter setting the compactness of supervoxels. The distance $\Upsilon$ is an iteratively calculated combination of Euclidean distance $d_{e}=\|\mathbf{x}-\mathbf{c}\|$ and the intensity-based similarity is $d_{I}=\|I(\mathbf{x})-I(\mathbf{c})\|$, where $\mathbf{x}$ is voxel position and $\mathbf{c}$ are cluster centers updated after each iteration. 

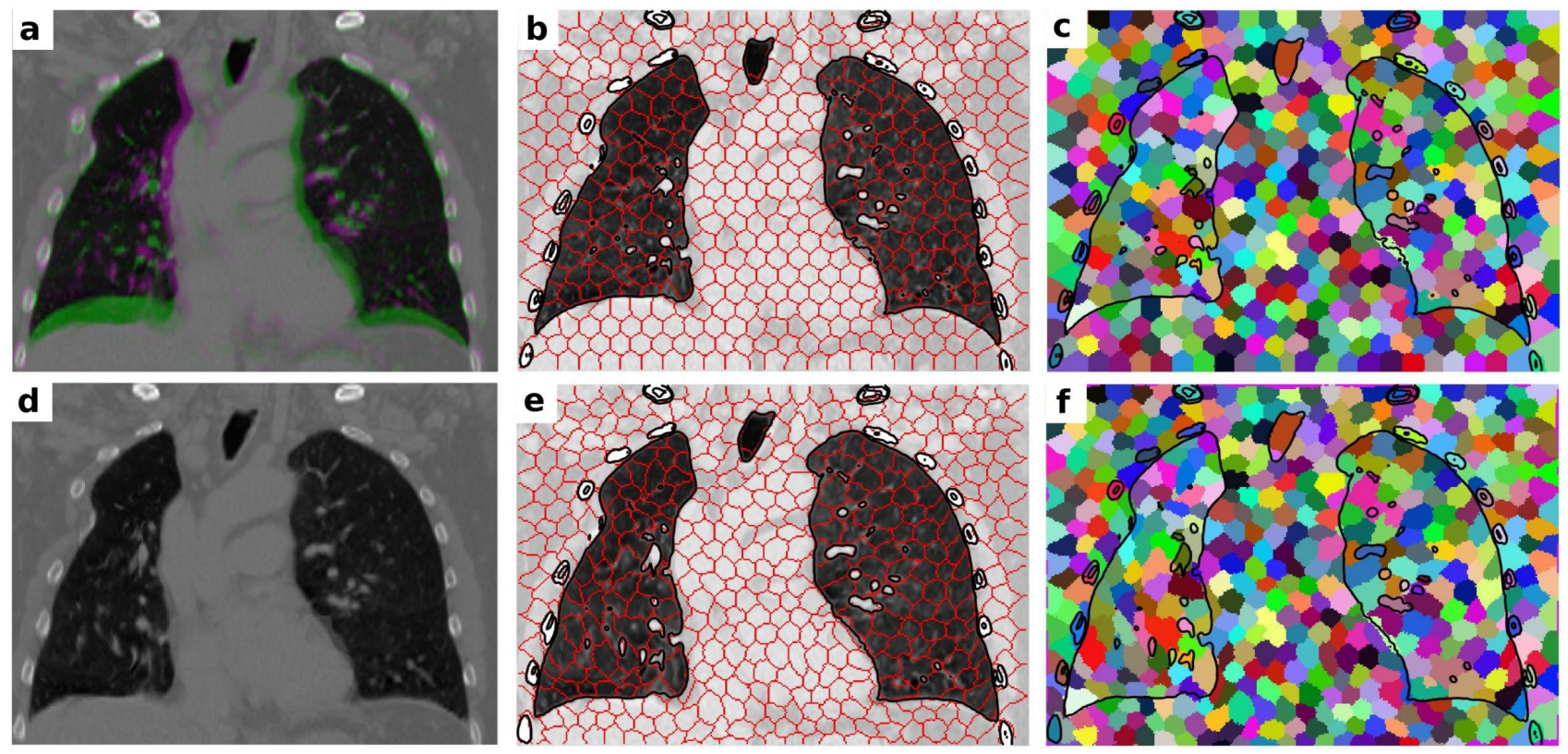

Figure 1. An example of the difference between peak exhale and peak inhale images (a), peak inhale image (d), SLIC clustering on peak exhale image (b,c) and their interpolation on peak inhale image (e,f). The original supervoxels extracted from peak exhale image are interpolated and maintain the same color. Contours on supervoxel images show lungs, ribs and main airways position. For illustrative purposes the supervoxels are extracted from a 2D image.

\subsection{Supervoxel Tracking for Ventilation Estimation}

In previous studies ventilation images were estimated for peak exhale ${ }^{8}$. However in our work we use peak inhale breathing phase as a reference, and therefore we formulate estimation of the ventilation for peak inhale as:

$$
V e n t_{D e n s}^{e x h}=\left(\frac{H U_{e x h}(\mathbf{x}) * G_{k 1}-H U_{i n h}\left(\mathbf{x}+\mathbf{u}_{i n h}\right) * G_{k 1}}{H U_{i n h}\left(\mathbf{x}+\mathbf{u}_{i n h}\right) * G_{k 1}+1000}\right) * G_{k 2},
$$

with $H U_{e x h}(\mathbf{x})$ standing for lung intensity value from peak exhale breathing phase image for voxel position $\mathbf{x}$ and $H U_{i n h}\left(\mathbf{x}+\mathbf{u}_{i n h}\right)$ lung intensity value from peak inhale breathing phase image for the same voxel moved by displacement field $\mathbf{u}_{i n h}$ and $G_{k 1}, G_{k 2}$ being Gaussian kernels convolved with the images. All the image intensities are given in HU.

We start by extracting supervoxels from the peak exhale breathing phase image and calculate their mean intensities. Then we apply the deformations originating from image registration between peak exhale and peak inhale breathing volumes to the extracted supervoxels. In this way we map the same regions, represented by supervoxels, from peak exhale to peak inhale image (see Fig. 1). We recalculate mean intensities of the transformed supervoxels, this time over the peak inhale image. Then we apply this approach to the method described above, thus estimating ventilation for supervoxels rather than in a voxel-wise manner:

$$
V e n t_{S L I C}=\frac{\overline{H U_{e x h}\left(\mathbf{s}+\mathbf{u}_{e x h}\right)}-\overline{H U_{i n h}(\mathbf{s})}}{\overline{H U_{i n h}(\mathbf{s})}+1000}
$$

where $\overline{H U_{i n h}(\mathbf{s})}$ is the mean intensity of the supervoxel extracted from peak inhale phase and $\overline{H U_{e x h}\left(\mathbf{s}+\mathbf{u}_{e x h}\right)}$ stands for mean intensity of the same supervoxel mapped to peak exhale breathing phase by deformation $\mathbf{u}_{e x h}$. Then all voxels belonging to the particular supervoxels are assigned the estimated ventilation value from Eq. 3.

In the approach of ${ }^{7,8}$ (referenced as "classic approach" from now on), described in Sec. 1, images are preprocessed using Gaussian filtering to compensate for mis-registration or other image artifacts. Therefore 

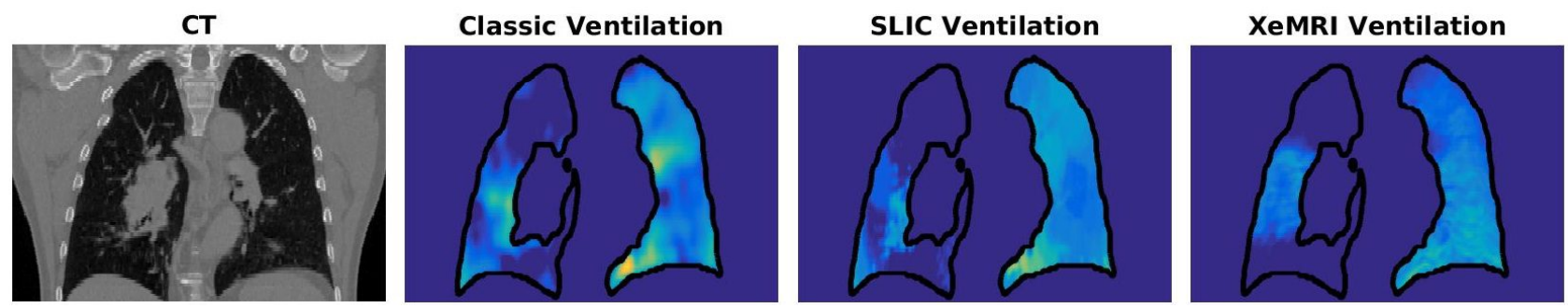

Figure 2. Comparison of the ventilation maps for Patient 2 in coronal view. Lung contours extracted from the CT image are propagated on ventilation images.

ventilation of each voxels is influenced by all of its neighbours in a certain spatial distance. As we propose to estimate the ventilation for groups of voxels which are spatially and visually close, such a formulation should more accurately correspond to the anatomical structure of the lungs.

To improve the robustness of the method, as well as to reduce the influence of the particular clustering, we propose to perform the extraction of a number of layers of supervoxels, akin to, ${ }^{12}$ where a similar approach has been applied to deformable image registration. Then the final estimated ventilation for a voxel is calculated as the average over all the layers:

$$
\overline{\operatorname{Vent}}_{S L I C}=\frac{1}{n} \sum_{1}^{n} \operatorname{Vent}_{S L I C}^{n}
$$

where $n$ is the number of layers of supervoxels. A visualization of the ventilation maps from both methods and XeMRI for Patient 2 is shown in Fig. 2. To further improve the results, we apply a small Gaussian filtering over the final $\overline{V e n t}_{S L I C}$. This could potential be omitted, if a large enough number of supervoxel layers was applied.

\section{EXPERIMENTS AND RESULTS}

We preformed the evaluation of the proposed method based on three cases consisting of 4DCT and XeMRI/pMRI data for patients undergoing radiotherapy treatment at Churchill Hospital in Oxford, using a number of criteria.

\subsection{Spatial Overlap Comparison}

We performed a series of experiments investigating the Dice overlap between ventilated and non-ventilated regions of the lungs. We started by evaluating the overlap between ventilated regions of the lungs for three percentile ranges of values: $70-100 \%, 80-100 \%$ and $90-100 \%$ of the ventilation volumes. Based on such clustering, we calculated the Dice overlap between them and XeMRI images clustered in the same manner. The achieved results for the classic approach are 0.806, 0.461, 0.335, whereas for the proposed approach they are 0.829, 0.460, 0.346 on average, respectively. The proposed method resulted in higher values of Dice overlap on average for all of the experiments, apart from the range of values $70-100 \%$, when compared to the classic approach. The numerical results are shown in Table 1.

We also performed clustering of non-ventilated parts of the lungs, based on three percentile ranges of values: $0-10 \%, 0-20 \%$ and $0-50 \%$ of the ventilation volumes. The results of the Dice overlap for these ranges are 0.415 , $0.459,0.653$ for the classic approach and $0.446,0.506,0.674$ for our method on average, respectively. For all of the experiments, the proposed method outperformed the classic approach resulting in higher values of Dice overlap on average. Table 2 presents the detailed results for each of the cases. 

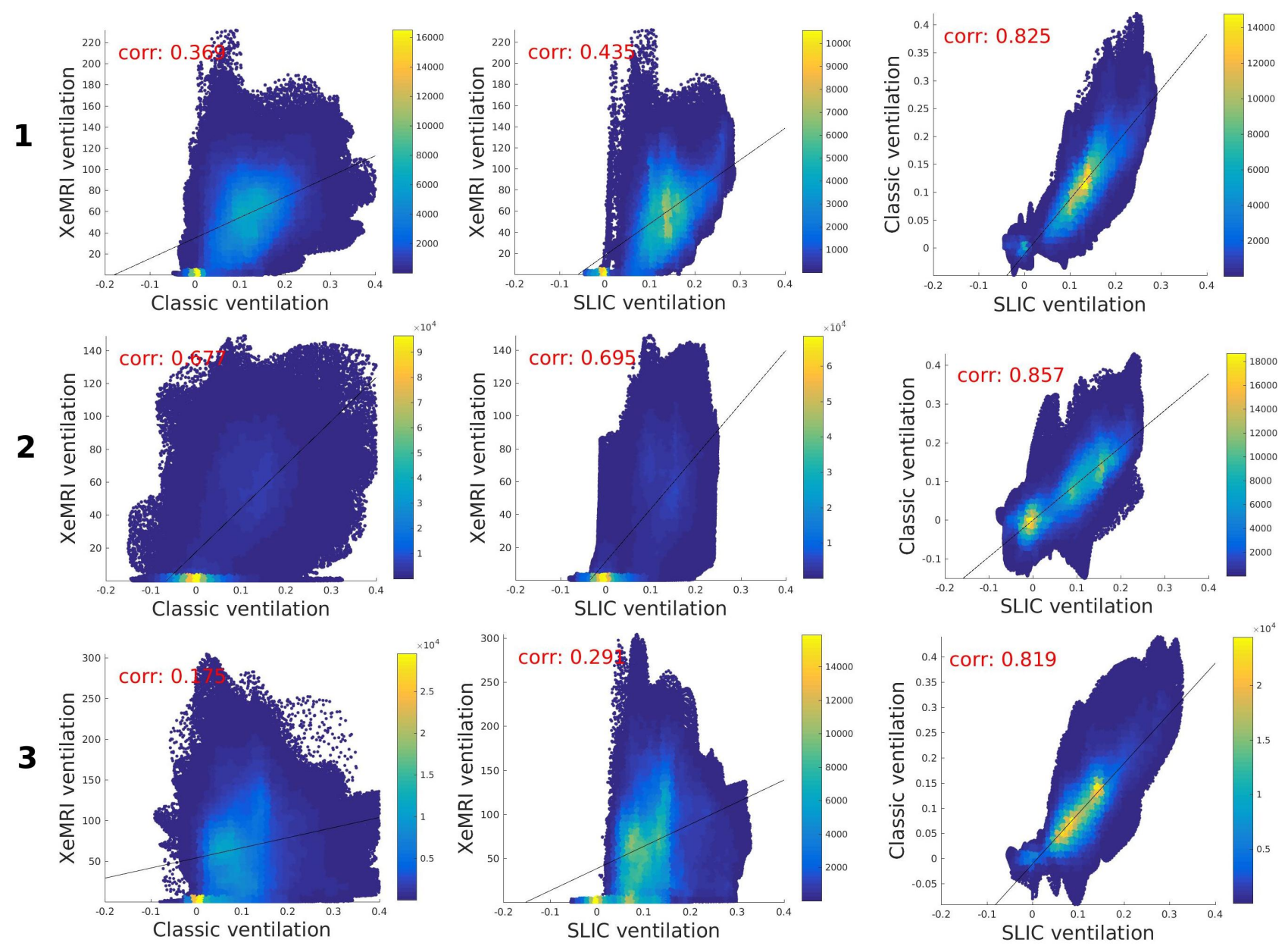

Figure 3. The figure shows correlation and Spearman correlation coefficient between the classic ventilation approach and XeMRI in the first column. In the second column, correlation and Spearman correlation coefficient between the proposed ventilation approach and XeMRI are presented. In the last column, correlation between the two ventilation estimation methods is shown. The best results can be observed for the proposed approach. Rows correspond to patient's number.

Table 1. Dice overlap calculated for ventilated regions of the lungs segmented from XeMRI, classic ventilation approach and our SLIC-based approach for different thresholds.

\begin{tabular}{c|c|c|c|c|c|c}
\hline & \multicolumn{2}{|c|}{$30-100 \%$} & \multicolumn{2}{c|}{$70-100 \%$} & \multicolumn{2}{c}{$80-100 \%$} \\
\hline Patient & Classic & SLIC & Classic & SLIC & Classic & SLIC \\
\hline 1 & 0.786 & $\mathbf{0 . 7 9 7}$ & 0.437 & $\mathbf{0 . 4 4 1}$ & 0.350 & $\mathbf{0 . 3 6 2}$ \\
2 & 0.895 & $\mathbf{0 . 9 2 4}$ & 0.530 & 0.483 & $\mathbf{0 . 3 7 5}$ & 0.341 \\
3 & 0.737 & $\mathbf{0 . 7 6 7}$ & 0.416 & $\mathbf{0 . 4 5 8}$ & 0.279 & $\mathbf{0 . 3 3 5}$ \\
\hline Avg & 0.806 & $\mathbf{0 . 8 2 9}$ & $\mathbf{0 . 4 6 1}$ & 0.460 & 0.355 & $\mathbf{0 . 3 4 6}$ \\
\hline
\end{tabular}


Table 2. Dice overlap coefficient calculated for non-ventilated regions of the lungs segmented from XeMRI, Classic ventilation approach and our SLIC-based approach for different thresholds.

\begin{tabular}{c|c|c|c|c|c|c}
\hline & \multicolumn{2}{|c|}{$0-10 \%$} & \multicolumn{2}{c|}{$0-20 \%$} & \multicolumn{2}{c}{$0-50 \%$} \\
\hline Patient & Classic & SLIC & Classic & SLIC & Classic & SLIC \\
\hline 1 & 0.485 & $\mathbf{0 . 5 3 3}$ & 0.466 & $\mathbf{0 . 5 0 3}$ & 0.614 & $\mathbf{0 . 6 3 0}$ \\
2 & 0.291 & $\mathbf{0 . 3 1 7}$ & 0.548 & $\mathbf{0 . 6 0 0}$ & $\mathbf{0 . 7 9 0}$ & 0.786 \\
3 & 0.470 & $\mathbf{0 . 4 8 9}$ & 0.364 & $\mathbf{0 . 4 1 6}$ & 0.557 & $\mathbf{0 . 6 0 6}$ \\
\hline Avg & 0.415 & $\mathbf{0 . 4 4 6}$ & 0.459 & $\mathbf{0 . 5 0 6}$ & 0.653 & $\mathbf{0 . 6 7 4}$ \\
\hline
\end{tabular}

\subsection{Global Correlation}

We calculate Spearman's correlation coefficient for both ventilation estimation methods: the classic intensity based and the proposed. We achieve higher correlation (0.487) compared to the commonly used method for estimating ventilation in a voxel-wise manner (0.423) on average for all cases. The visualization of the results is presented in Fig. 3 for all the cases. The numerical results are presented in Table 3. We also calculate correlation between both ventilation estimation methods, showing that they correlate well (0.834), yet still our method gives higher correlations with XeMRI.

Table 3. Pearson's and Spearman's correlation coefficients calculated for the classic and our SLIC-based ventilation estimation approach for 15 layers of supervoxels.

\begin{tabular}{c|c|c|c|c}
\hline & \multicolumn{2}{|c|}{ Classic } & \multicolumn{2}{c}{ SLIC } \\
\hline Patient & Pearson & Spearman & Pearson & Spearman \\
\hline 1 & 0.395 & 0.368 & $\mathbf{0 . 5 0 7}$ & $\mathbf{0 . 4 3 5}$ \\
2 & 0.646 & 0.677 & $\mathbf{0 . 6 9 7}$ & $\mathbf{0 . 6 9 5}$ \\
3 & 0.175 & 0.224 & $\mathbf{0 . 2 9 1}$ & $\mathbf{0 . 3 3 1}$ \\
\hline Avg & 0.405 & 0.423 & $\mathbf{0 . 4 9 8}$ & $\mathbf{0 . 4 8 7}$ \\
\hline
\end{tabular}

\subsection{Influence of the Number of Layers}

The number of layers of supervoxels has an obvious influence on the estimated ventilation images. The results of the conducted experiment range from 0.393 for just one layer, 0.463 for 5 layers, to 0.487 for 15 layers on average for Spearman's correlation coefficient. The detailed results for each individual case are presented in Table 4 . This shows that a higher number of layers of supervoxels provides images, which give higher correlation with XeMRI. As it was expected, a higher number of layers of supervoxels improves the robustness of the method for mis-segmentations originating from a particular supervoxels extraction.

Table 4. Comparison of Pearson's and Spearman's correlation coefficients calculated for the SLIC-based ventilation estimation approach for 1,5 and 15 layers of supervoxels

\begin{tabular}{c|c|c|c|c|c|c}
\hline & \multicolumn{2}{|c|}{ 1 layer } & \multicolumn{2}{c|}{5 layers } & \multicolumn{2}{c}{15 layers } \\
\hline Patient & Pearson & Spearman & Pearson & Spearman & Pearson & Spearman \\
\hline 1 & 0.451 & 0.365 & 0.460 & 0.397 & $\mathbf{0 . 5 0 7}$ & $\mathbf{0 . 4 3 5}$ \\
2 & 0.628 & 0.621 & 0.658 & 0.675 & $\mathbf{0 . 6 9 7}$ & $\mathbf{0 . 6 9 5}$ \\
3 & 0.197 & 0.194 & 0.262 & 0.319 & $\mathbf{0 . 2 9 1}$ & $\mathbf{0 . 3 3 1}$ \\
\hline Avg & 0.425 & 0.393 & 0.460 & 0.463 & $\mathbf{0 . 4 9 8}$ & $\mathbf{0 . 4 8 7}$ \\
\hline
\end{tabular}




\section{CONCLUSIONS}

We have shown that our proposed method achieves higher correlation coefficients compared to the classic approach when correlated with XeMRI. A detailed analysis including a larger group of patients could further support our results. The presented results, where for majority of the conducted experiments the proposed method for ventilation estimation from 4DCT achieves better correlations with XeMRI images, indicate higher physiological consistency of our proposed approach using supervoxels for ventilation estimation. In the future, we are planning to perform further analysis on a larger patient group and investigate the influence of different deformable image registration methods on the correlation, especially those that explicitly address sliding motion in the lungs during breathing. Another crucial issue is XeMRI to CT registration, which we are planning to further enhance for more accurate results.

\section{ACKNOWLEDGMENTS}

We would like to acknowledge funding from the CRUK \& EPSRC Cancer Imaging Centre in Oxford. We would also like to acknowledge M. Heinrich for making his code for deeds ${ }^{9}$ available online.

\section{REFERENCES}

[1] Murray, C.J.L., "Global, regional, and national age-sex specific all-cause and cause-specific mortality for 240 causes of death, 1990-2013: a systematic analysis for the Global Burden of Disease Study 2013", Lancet. Jan 10, 117-171 (2015)

[2] Ferlay, J., Soerjomataram, I., Ervik, M., Dikshit, R., Eser, S., Mathers, C., Rebelo, M., Parkin, D.M., Forman, D., Bray, F., "Cancer Incidence and Mortality Worldwide: IARC CancerBase 11", International Agency for Research on Cancer, (2014)

[3] Albert, M.S., Cates, G.D., Driehuys, B., Happer, W., Saam, B., Springer, C.S.Jr, Wishnia, A., "Biological magnetic resonance imaging using laser-polarized 129Xe", Nature 370, 199-201 (1994)

[4] Mugler, J.P., Altes, T.A., "Hyperpolarized 129Xe MRI of the human lung.", J Magn Reson Imaging 37(2), 313-331 (2013)

[5] Reinhardt, J.M., Ding, K., Cao, K., Christensen, G.E., Hoffman, E.A., Bodas, Sh.V., "Registration-based estimates of local lung tissue expansion compared to xenon-CT measures of specific ventilation", Med Image Anal. 12(6), 724-32 (2008)

[6] Yamamoto, T., Kabus, S., Klinder, T., Lorenz, C., von Berg, J., Blaffert, T., Loo, B.W., Keall,, P.J., "Investigation of four-dimensional computed tomography-based pulmonary ventilation imaging in patients with emphysematous lung regions", Phys Med Biol 56(7), 2279-98 (2011)

[7] Guerrero, T., Sanders, K., Noyola-Martinez, J., Castillo, E., Zhang, Y., Tapiaa, R., Guerra, R., Borghero, Y. Komaki, R., "Quantification of regional ventilation from treatment planning CT", Int J Radiat Oncol Biol Phys 62(3), 630-34 (2005)

[8] Yamamoto, T., Kabus, S., Klinder, T., von Berg, J., Lorenz, C., Loo, B. W., Keall, P.J., "Four-dimensional computed tomography pulmonary ventilation images vary with deformable image registration algorithms and metrics", Med Phys 38, 1348-58 (2011)

[9] Heinrich, M.P., Jenkinson, M., Brady, M., Schnabel, J.A., "MRF-based deformable registration and ventilation estimation of lung CT", IEEE Trans Med Imag 32(7), 1239-48 (2013)

[10] Murphy, K., van Ginneken, B., Reinhardt, J.M. et al., "Evaluation of registration methods on thoracic CT: the EMPIRE10 challenge", IEEE Trans Med Imag 30(11), 1901-20 (2011)

[11] Achanta, R., Shaji, A., Smith, K., Lucchi, A., Fua, P., Süsstrunk, S., "SLIC superpixels compared to state-of-the-art superpixel methods", IEEE Trans Pattern Anal Mach Intell 34(11), 2274-82 (2012)

[12] Heinrich, M.P., Simpson, I.J.A., Papież, B.W., Brady, M., Schnabel, J.A., "Deformable image registration by combining uncertainty estimates from supervoxel belief propagation", Med Image Anal 27, 57-71 (2016) 